Cita bibliográfica: Precedo Ledo, A., y Míguez Iglesias, A. (2018). Los efectos de la crisis en el posicionamiento de las ciudades españolas. Boletín de la Asociación de Geógrafos Españoles, 76, 79-101. doi: $10.21138 /$ bage. 2516

\title{
Los efectos de la crisis en el posicionamiento de las ciudades españolas
}

The effects of the crisis in the positioning of Spanish cities

\author{
Andrés Precedo Ledo \\ andresjose.precedo@usc.es \\ Departamento de Geografía \\ Universidad de Santiago de Compostela (España) \\ Alberto Míguez Iglesias \\ amiguez@escuni.com \\ Departamento de CC.SS. y Sociología \\ Centro Universitario de Magisterio Escuni (España)
}

\section{Resumen}

Esta investigación tiene por objeto hacer una medición empírica del impacto de la crisis económica (2007-2014) en los cambios de posicionamiento y en la resiliencia de las 22 ciudades españolas con mayor peso demográfico y económico en la fase de salida. Los principales objetivos son cuatro: medir los efectos de la crisis económica y de la burbuja inmobiliaria en los cambios de posicionamiento en el sistema metropolitano; estimar el diferente peso desempeñado por las iniciativas económicas del sector privado iniciativa privada y las políticas del sector público; evaluar la eficacia de las políticas estratégicas y de marketing urbano en el reposicionamiento de las ciudades; y determinar el perfil emprendedor de la economía urbana como factor de resiliencia y de potenciación del desarrollo urbano.

Palabras clave: crisis económica; resiliencia; posicionamiento urbano; políticas de economía urbana. 


\begin{abstract}
This research is aimed at empirically measuring the impact of the economic crisis (2007-2014) on the changes of positioning and on the resilience of the 22 Spanish cities with a greater demographic and economic weight in the starting line phase. The main objectives are four: to quantify the effects of the economic crisis and the real estate bubble on the changes of positioning in the metropolitan system: to assess the different weight taken by economic initiatives in the private sector, private initiative, and the policies of the public sector: to evaluate the efficacy of strategic and urban marketing policies in the repositioning of cities: and to determine the entrepreneurial profile of urban economy as a factor of resilience and strengthening of urban development.
\end{abstract}

Key words: economic crisis; resilience; positioning of the city; urban economy policy.

\title{
1 Introducción
}

Son muchos los trabajos realizados en los últimos años sobre los efectos territoriales de la crisis económica en España. La mayoría de ellos toman como referencia la burbuja inmobiliaria (Miguel, 2009; Gentier, 2012; Vinuesa, 2013), por ser el factor esencial en el crecimiento expansivo y posterior reversión de las ciudades, y en la evolución urbana del crecimiento económico y del empleo. Las políticas urbanas aplicadas en la etapa anterior (Burriel, 2008) aceleraron los ya importantes desajustes del proceso de urbanización, y posteriormente dieron lugar a una crisis múltiple que se manifestó en todos los factores asociados al desarrollo territorial. Al mismo tiempo, y por razones concomitantes, la excesiva carga hipotecaria afectó negativamente a muchas economías familiares y a la mayoría de las entidades bancarias. Todo esto, unido a los efectos de la crisis financiera internacional, provocó un reajuste del sistema bancario que arrastró el desplome de muchas economías locales y generó un aumento del ya insostenible déficit público, y que al final se saldó con un fuerte endeudamiento de las haciendas locales que se estaban financiando con los beneficios derivados de la promoción inmobiliaria. Un proceso que, de manera acumulativa, desestabilizó el propio presupuesto nacional por el rescate bancario pagado con fondos públicos en el marco de una política económica neoliberal. Unos datos sirven para demostrar la importancia que tuvo la burbuja inmobiliaria: la evolución de la promoción de viviendas en el periodo 20072012 (Burriel, 2008): si en 2007 se inició la construcción de 1149 viviendas nuevas, en 2012 el número había bajado a tan solo 67 045; y si en el primer año de las 647179 viviendas terminadas las de promoción pública eran solo el 10,4\%, en el último esta proporción aumentó hasta el 40 \%, sobre un total de 133425 viviendas.

Estas cifras son elocuentes de lo que ha ocurrido y de sus consecuencias en el crecimiento urbano, principalmente de las zonas turísticas que, junto con los desarrollos de las periferias metropolitanas (Méndez, 2013) fue donde la burbuja inmobiliaria alcanzó su mayor amplitud. Una caída de tales dimensiones generó una serie de efectos encadenados: el exceso del parque de viviendas 
construidas, el desplome del mercado inmobiliario y la crisis hipotecaria de un sistema bancario que había actuado como facilitador, la pérdida de empleo en la construcción que había sido el motor de la atracción de fuertes corrientes migratorias que entraron en un prolongado paro estructural, un exceso del stock de suelo e incluso de suelo urbanizado, la ocupación intensiva de suelos productivos por una urbanización de baja densidad fuertemente consumidora de suelo y de recursos naturales, y, al mismo tiempo, un desajuste entre las políticas y los instrumentos de planificación urbana y la nueva realidad que afectó al núcleo mismo del proceso de urbanización (Ramírez, 2010). A ello se sumó que a las rentas derivadas de ese crecimiento descontrolado del sector de la construcción se añadió el crecimiento de sectores industriales y de servicios asociados, que aportaron importantes ingresos en los presupuesto de las familias y de los gobiernos locales, lo que llevó a un incremento exponencial del gasto público en políticas urbanísticas despilfarradoras (Burriel, 2008) y en el consumo interno de las familias. En definitiva, un modelo económico extremadamente vulnerable que, al entrar en crisis arrastró consigo a la mayoría de los factores de producción. Como consecuencia, entre 2007 y 2012 todo el sistema económico y social se vino abajo, coincidiendo con la fase álgida de la crisis financiera internacional.

Una situación como la descrita habría de afectar necesariamente a las ciudades españolas, siendo mayor el impacto allí donde la burbuja había generado los mayores crecimientos, y que fue donde los problemas sectoriales y del empleo fueron también más ostensibles y donde la imagen de las grandes promociones y urbanizaciones inacabadas entraron a formar parte del paisaje urbano español de la primera década del siglo actual (Vinuesa, y Martin, 2013; Méndez y Plaza, 2016). Como bien demostró Méndez (Atlas de la Crisis, 2013), los principales efectos negativos se produjeron en las ciudades y provincias del litoral mediterráneo, principalmente el tramo levantino y almeriense, y Canarias, es decir allí donde se concentró la demanda turística, la oferta de segunda vivienda vacacional (que atrajo compradores con rentas de trabajo crecientes cautivados por la política hipotecaría de los bancos) y las promociones orientadas a compradores extranjeros. Allí también se habían acumulado los inmigrantes (Vono y Ballona; 2010) y fue también allí donde se pusieron en marcha políticas de gasto en actuaciones desmesuradas de urbanismo de marketing, promovidas por algunos municipios y por las propias Comunidades Autónomas (Comunidad Valenciana, por ejemplo) (Del Romero, 2010). Tal acumulación de factores explica perfectamente las dimensiones del problema en esas zonas. Por el contrario, las provincias donde la base económica estaba más centrada en actividades productivas exportadoras que en el turismo, y con empleos de mayor calidad, el efecto de la burbuja fue menos notable, como ocurrió en el Bilbao, San Sebastián, Vitoria, Pamplona o en La Coruña (Campos y Navarro, 2013), y en las provincias donde la economía urbana tiene como base la industria del automóvil ocurrió lo mismo (Palencia, Vitoria, Pamplona Zaragoza, Pontevedra, Valladolid). Mientras, en las provincias con una economía más rural y que estuvieron al margen de los factores citados del reciente proceso de urbanización, 
el efecto fue mucho menor (interior de Galicia, Zamora y Extremadura en la frontera portuguesa). Un último aspecto que se puede añadir, siguiendo los datos aportados por estos estudios, es el efecto generado por la descentralización del crecimiento de Madrid en las provincias de su entorno, y en particular allí donde la construcción de nuevos tramos del AVE generó expectativas para el sector inmobiliario (Toledo y Segovia, por ejemplo). Esta urbanización creciente de las periferias metropolitanas en base a desarrollos de baja densidad tuvo lugar en todas las ciudades, dando lugar a un urbanismo poco o nada sostenible, que añadiría sus efectos a los anteriores.

Como al principio dijimos, este fue el marco en el que se desarrollaron la mayoría de los estudios realizados, y que quedaron magníficamente resumidos por Méndez (2013) en su libro "Las escalas de la crisis". A nosotros nos pareció interesante centrarnos en un estudio comparativo de las principales áreas urbanas desde el punto de vista económico, con el fin de averiguar las posibles relaciones existentes entre la crisis económica general y las políticas urbanas aplicadas en la etapa anterior (planificación estratégica, marketing urbano), de un lado, y los factores asociados a la nueva industrialización y a las actividades empresariales más exportadoras, de otro. La selección de ciudades la hemos hecho con una discriminación positiva de lo que suele llamarse "el efecto sede", es decir la importancia que pueda tener este hecho en el desarrollo económico de las ciudades donde radican, de ahí la introducción del criterio empresarial en la selección de las ciudades. Así como el análisis de los resultados de la burbuja inmobiliaria tuvo muchos efectos comunes a todas las ciudades, también el ajuste del sistema productivo, que tuvo que reinventarse en su casi totalidad, tuvo como efecto común la rápida apertura al mercado global para compensar los efectos de la caída de la demanda interna y para adaptarse a los nuevos modelos mundiales de competitividad. En un primer momento fueron las mayores empresas españolas las que, buscando una salida a las nuevas condiciones del mercado, orientaron su actividad a los mercados exteriores insertándose en los flujos económicos propios de la globalización. En las economías urbanas más evolucionadas, como en las ciudades vascas, este proceso de internacionalización se inició también en las pequeñas y medianas empresa. Este hecho compensó, de alguna manera, los efectos negativos de la crisis inmobiliaria en las ciudades donde se localizaban las grandes empresas y las actividades de la nueva economía, por cuanto la expansión del negocio estuvo acompañada de un incremento y la reinversión de parte de los beneficios en la creación de empleos de calidad que crearon una demanda urbana más robusta. Un efecto posterior fue el que tuvieron en el desarrollo de nuevos servicios profesionales y de aplicaciones avanzadas de software que dieron lugar a un nuevo sector terciario creativo, así como a la formación de un nuevo tejido productivo destinado al aprovisionamiento de las grandes empresas. Tal vez el ejemplo más significativo sea el caso Inditex que creó en torno a su sede un efecto inducido que se concretó en la creación o fortalecimiento de una red de 1100 empresas proveedoras y la creación de 30000 empleos añadidos a los existentes durante el periodo analizado, y que al mismo tiempo les facilitó la apertura a los nuevos 
mercados en donde estaban actuando, aportando como efecto innovador la internacionalización del tejido productivo local. Por eso hemos pensado en diferenciar los efectos ligados a la crisis económica e inmobiliaria, que afectaron duramente a las iniciativas públicas y privadas presentes en el proceso de urbanización, de los efectos positivos generados por las empresas mayores que se adaptaron con éxito a las nuevas condiciones del sistema económico, acrecentando la exportación de su producción y la penetración en nuevos mercados, donde llegaron a alcanzar posiciones de liderazgo, como ocurrió con Iberdrola-Gamesa en el campo de las energías renovables. Sin embargo, las estadísticas existentes no permitían establecer esa disociación de factores, por lo cual acudimos a la actualización de un cuestionario que habíamos elaborado para las ciudades españolas mayores de 200000 habitantes donde, además, se localizaban las sedes de las cien mayores empresas españolas. Estos fueron los puntos de partida de este trabajo, y aunque somos conscientes de sus limitaciones, creemos pueden aportar una nueva visión al problema estudiado.

\section{Objetivos}

Los objetivos de nuestro estudio fueron varios. En primer lugar, medir los efectos que la crisis económica y la burbuja inmobiliaria han tenido en el posicionamiento de las ciudades españolas seleccionadas, estableciendo una comparativa entre el posicionamiento de entrada y el de salida. Un segundo objetivo era distinguir el diferente peso, que los casos de posicionamiento han tenido en la iniciativa pública y la iniciativa empresarial, otorgándole un papel esencial al efecto sede de las grandes empresas multinacionales. En tercer lugar nos propusimos evaluar, de manera indirecta, la eficacia de las políticas estratégicas y de marketing urbano que las ciudades seleccionadas habían desarrollado en mayor o menor medida. Un último punto fue determinar el carácter emprendedor de las ciudades como factor potenciador del desarrollo urbano.

\section{Metodología y fuentes}

El punto de partida ha sido un cuestionario realizado a expertos universitarios en geografía y economía urbana de las respectivas universidades, cuyos contenidos y resultados han sido objeto de una descripción pormenorizada anterior (Precedo y Míguez, 2014).

El cuestionario se articula en cuatro partes: estructura urbana y organización espacial, actuaciones urbanas, economía urbana y jerarquía urbano, y reputación ciudad. El número de ciudades estudiadas ha sido de 22 áreas metropolitanas, seleccionadas en función de su población (más de 200000 habitantes en el municipio, INE 2012) y su peso económico y empresarial, que en el área metropolitana se localice alguna de las sedes centrales y grandes unidades de producción de las 500 mayores empresas españolas (Expansión 2011).

A partir de la información obtenida, aplicamos la siguiente metodología: 
1. Cuantificamos las respuestas del cuestionario mediante una primera ponderación según fueran respuestas cerradas, abiertas o en rangos.

2. Para cuantificar el peso de cada ciudad, homogeneizamos los valores ponderados obtenidos, y así calculamos el sumatorio final.

Figura 1. Mapa de distribución de las ciudades diagnosticadas

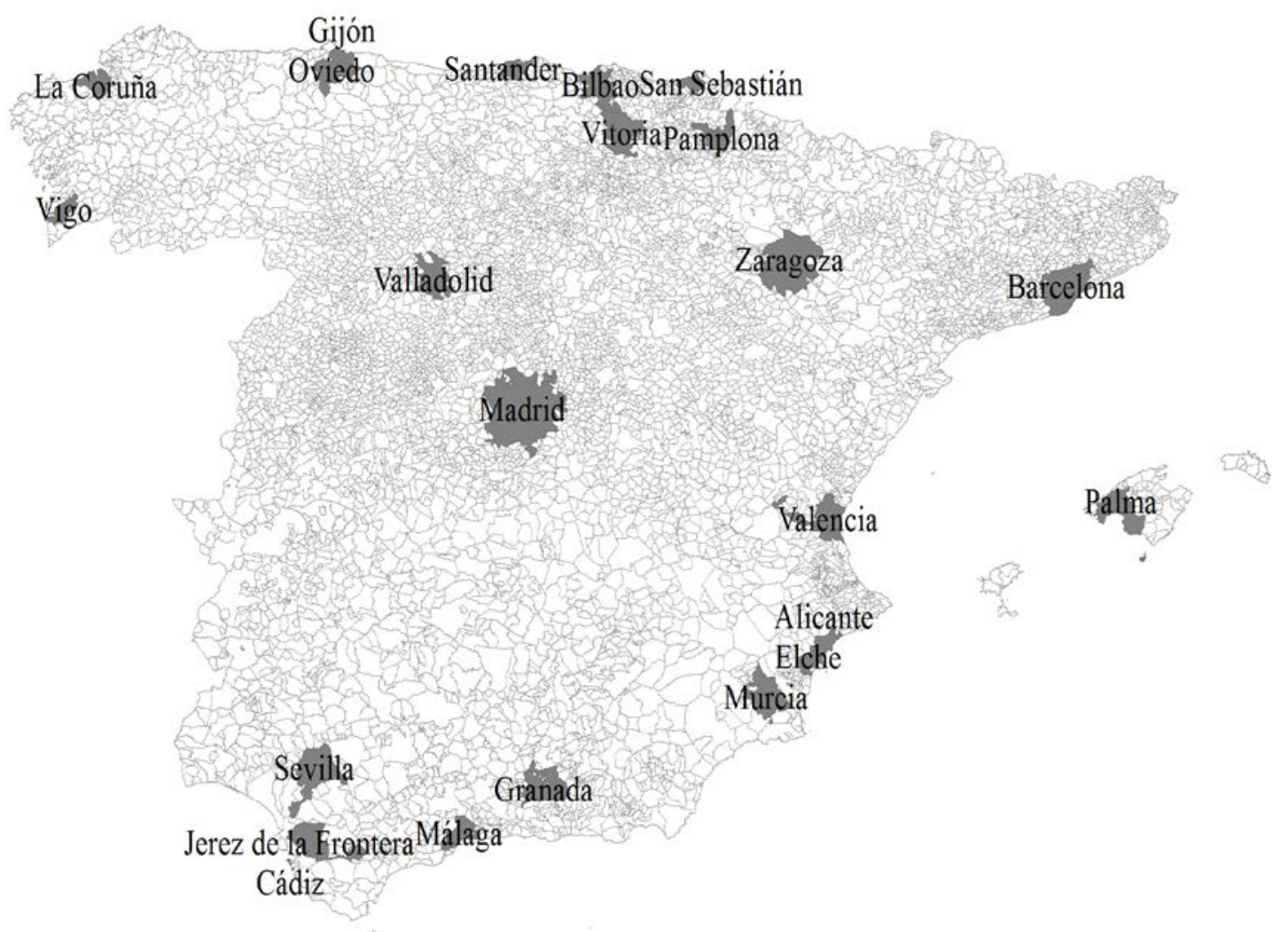

Fuente: Atlas estadístico de las áreas urbanas de España (2012)

3. Una vez obtenida una base numérica homogénea, procedimos a calcular el valor de cada una de las variables y las agrupamos según los dos tipos de iniciativas predominantes (pública y privada), de acuerdo con los objetivos de la investigación. Así, calculamos el índice que mide el peso de las iniciativas privadas o públicas en cada ciudad, según la clasificación que en su momento detallaremos. De este modo, obtuvimos el valor numérico de cada factor, y con la suma de ambos calculamos un índice de posicionamiento de cada ciudad, lo que nos permitió medir el valor de la correlación estadística entre los dos factores generales y el índice final de posicionamiento, para averiguar si había algún resultado significativo.

4. El siguiente paso fue calcular el índice privado/público, dividiendo los índices parciales anteriores. Cuando el valor supera la unidad quiere decir que en esa ciudad el principal factor ha sido el privado. Obtuvimos así el ranking de las ciudades según el peso de la iniciativa privada en relación con la pública, y esto nos permitió clasificar las ciudades según el peso 
numérico de las iniciativas vinculadas principalmente con la gestión privada en relación con las de carácter exclusivamente público.

5. Un segundo nivel de análisis, se centró en la medición de la situación de salida de cada ciudad al inicio de la salida de la fase recesiva de la crisis. Hicimos una doble medición.

a) Un índice evolutivo para medir el cambio de cada ciudad, a partir de las variables que a continuación enumeramos (los datos para este indicador fueron obtenidos del INE, Seguridad Social y Anuario La Caixa para el periodo 2006-2014):

i. Incremento de pobl. 07/14

ii. Variación paro registrado 07/14

iii. Variación Contratos indefinidos 07/14

iv. Variación Índice actividad económica 06-11

La clasificación resultante nos permitió conocer el crecimiento relativo de la economía urbana, es decir las ciudades que crecieron más o menos, pero no su posicionamiento estructural de salida. Así por ejemplo, Elche, ocupa puestos muy elevados en la tabla evolutiva a pesar de mantener una posición baja en el posicionamiento urbano, debido a tener valores negativos o inferiores a la media, pero como el punto de partida era muy bajo y en el periodo experimentó fuertes inputs, la ciudad advirtió una fuerte mejora.

b) Un índice estructural, tomando los datos de Urban Audit e INE (periodo 2007-14). Este es el que adoptamos como índice de desarrollo urbano de cada ciudad y que mide la respuesta a la salida de la crisis. Las variables utilizadas fueron:

1) Incremento de población $07 / 14$

2) Variación paro registrado $07 / 14$

3) Variación Contratos indefinidos 07/14

4) Variación Índice actividad económica 06-11

5) Proporción de extranjeros sobre la población total (Porcentaje)

6) Tasa de desempleo (Porcentaje)

7) Proporción de ocupados entre 20-64 años sobre la población activa (Porcentaje)

8) Tasa de actividad (Porcentaje)

9) Proporción de empleo en servicios (NACE Rev.2 G-U) (Porcentaje)

10) Proporción de empleo en industria (NACE Rev.2 B-E) (Porcentaje) 
11) Renta neta media anual de los hogares (Euros)

12) Proporción de empresas del sector industrial sobre el número total de empresas (Porcentaje)

De este modo, intentamos obtener una respuesta al segundo objetivo del estudio: estimar el posicionamiento de las ciudades después del período recesivo de la crisis, y averiguar cuáles fueron las causas principales de los cambios.

6. Finalmente, calculamos las correlaciones existentes entre ambas clasificaciones y el índice privado/público, y, para reforzar estas conclusiones hicimos una última medición: calculamos el coeficiente de correlación entre el índice de situación y el índice privado y el público por separado. Los resultados se exponen a continuación.

\section{Los factores e índice del posicionamiento urbano}

Para calcular el índice de posicionamiento inicial de las ciudades (2007-2012), y para agrupar los indicadores numéricos obtenidos tras la cuantificación del cuestionario citado, en función de tipo de iniciativa predominante, hemos analizado 21 ítems, y de ellos hemos seleccionado 14 como los más significativos y los que aportan un dato numérico más objetivable. Estos indicadores los describimos a continuación agrupados en los dos tipos de iniciativa establecidos. En cada caso enumeraremos las ciudades mejor y peor posicionadas. Son los siguientes:

A. Acciones predominantemente dependientes de la iniciativa privada:

1. Gestión empresarial. Madrid, Bilbao, Valencia, La Coruña

2. Competitividad económica. Madrid, La Coruña, Bilbao, Pamplona.

3. Mejora del posicionamiento económico-empresarial. Madrid, Valencia, Bilbao, Málaga, La Coruña.

4. Diversificación de la base económica urbana. Barcelona, Madrid y La Coruña

5. Globalización/internacionalización empresas. Madrid, Bilbao, La Coruña

6. Oferta empresas turísticas (renovación oferta hoteles). Barcelona, Madrid, Palma, Bilbao, Granada, Málaga y Pamplona

7. Rango cuota comercial de mercado. Madrid, Barcelona, Bilbao y Vigo

Desde el punto de vista económico empresarial, Madrid es la ciudad española que ocupa siempre el primer lugar, perdiendo posiciones Barcelona a favor de Valencia, mientras en el Norte, La Coruña, por el efecto Inditex-Zara, se equipara o supera ligeramente a Billbao. Un resultado concordante con otros estudios realizados sobre este particular. Como referente podemos citar la última edición del ranking de ciudades mundiales Cities in Motion, elaborado por el I.E.S.E, en el 
cual la ciudad gallega ocupa el cuarto lugar entre las españolas. El resultado quedó así: Madrid (con un perfil empresarial dominante), Barcelona (con un perfil turístico y empresarial), Valencia (comercio y turismo), La Coruña por el "efecto sede" del primer grupo empresarial español, y en cuarto lugar Bilbao (empresarial).

B. Acciones predominantemente dependientes del sector público:

1. Gestión metropolitana. Barcelona, Sevilla, Oviedo-Gijón-Avilés y San Sebastián

2. Planificación estratégica área metropolitana. Barcelona, Málaga y San Sebastián

3. Dinámica expansiva crecimiento espacial. Madrid, Barcelona, Málaga, Bahía de Cádiz y S. Sebastián

4. Calidad de vida urbana. Oviedo y San Sebastián, Pamplona y Bilbao

5. Operaciones de renovación e innovación urbana. Barcelona, Bilbao y Valencia

6. Promoción y marketing exterior. Barcelona y en segunda posición Valencia y Bilbao

7. Venta producto turístico global. Barcelona

Desde el punto de vista de la mejor gestión institucional de las ciudades, que se traduce en una mayor calidad de vida y un urbanismo más innovador, destaca siempre Barcelona, donde se realizó la mayor operación de renovación urbana y de marketing territorial de España, con un efecto reputacional muy relevante a nivel mundial. Por razones parecidas Valencia y Málaga, se constituyeron en ciudades de referencia en políticas de promoción y marketing urbano; seguidas de las ciudades del Norte, donde destaca Bilbao, probablemente el caso de éxito más relevante de marketing urbano. También la metrópoli vasca destaca por el nivel de excelencia del diseño urbano, y por la calidad de vida; pero en este aspecto las ciudades que más destacan son, respectivamente, Pamplona, San Sebastián, Vitoria y Oviedo, valoradas como las mejores ciudades españolas en todos los ranking que tienen en cuenta el urbanismo, la movilidad y la sostenibilidad. Estos resultados avalan, de alguna manera, el resultado positivo o de impulso que supusieron los planes de renovación urbana efectuado en las ciudades de Barcelona, Valencia, Bilbao y Málaga, sin duda los más ambiciosos de los realizados en España, aunque en algunos casos, otros factores relacionados con la gestión cuestionan la validez de los resultados (Valencia por ej., pero el efecto impulsor inicial fue incuestionable.

El último aspecto a considerar es el relativo al índice de posicionamiento para el período, obtenido mediante la suma de los dos índices sectoriales o parciales comentados. El resultado se expone en la siguiente tabla: 
Tabla 1. Índice de posicionamiento

\begin{tabular}{|l|r|r|r|r|}
\hline Ciudad & PRIVADO & PÚBLICO & TOTAL & Grupo \\
\hline Billbao & 54,4 & 68,9 & 123,3 & I \\
\hline Madrid & 56,5 & 64,6 & 121,1 & I \\
\hline Barcelona & 53,4 & 65,4 & 118,8 & I \\
\hline La Coruña & 55,8 & 47,9 & 103,7 & I \\
\hline Valencia & 46,0 & 57,3 & 103,3 & I \\
\hline Málaga & 47,6 & 49,7 & 97,3 & II \\
\hline San Sebastián & 35,6 & 57,5 & 93,6 & II \\
\hline Pamplona & 38,2 & 51,2 & 89,4 & II \\
\hline Oviedo (Ciudad Astur) & 34,2 & 52,6 & 86,8 & II \\
\hline Sevilla & 35,2 & 46,4 & 81,6 & II \\
\hline Vigo & 37,3 & 42,2 & 79,5 & III \\
\hline Zaragoza & 34,0 & 43,5 & 77,5 & III \\
\hline Palma de Mallorca & 36,0 & 35,4 & 71,4 & III \\
\hline Valladolid & 28,3 & 41,3 & 69,6 & III \\
\hline Granada & 28,1 & 39,9 & 68,0 & III \\
\hline Vitoria & 25,7 & 35,3 & 61,0 & III \\
\hline Murcia & 21,6 & 35,6 & 57,2 & IV \\
\hline Santander & 23,1 & 33,6 & 56,7 & IV \\
\hline Alicante & 21,0 & 33,6 & 54,6 & IV \\
\hline Jerez & 14,2 & 36,0 & 50,2 & IV \\
\hline Cádiz & 17,6 & 29,7 & 47,3 & IV \\
\hline Elche & 20,3 & 25,7 & 46,0 & IV \\
\hline
\end{tabular}

Fuente: elaboración propia

Para su clasificación, y después de varios intentos, hicimos una jerarquización en rangos por cuartiles, calculados según una aproximación a los intervalos inferior (40) y superior (100). El resultado presenta una interesante secuencia:

- Clase I (más de 100): Bilbao y Madrid son las dos ciudades con mejor posicionamiento en las variables consideradas, y a ellas añadimos Barcelona, La Coruña y Valencia, aunque su valor numérico queden por debajo. Bilbao es, con Barcelona, la ciudad donde la combinación del impulso empresarial y la intensa renovación urbana, asociados a una estrategia ambiciosa de marketing urbano, ha dado mejores resultados, pero Madrid se ha convertido en el principal centro mundial de decisiones y de negocios de España. Por eso, el perfil funcional de cada una es diferente según el valor de las variables analizadas: Madrid es ya una ciudad global desde el punto de vista financiero y empresarial, Barcelona lo es en el plano turístico tras una pérdida de su anterior peso económico, y Bilbao se apoya en el peso de una gestión urbana de éxito a nivel internacional. Con todo, son las ciudades más destacadas en todas las variables. El segundo grupo está formado por dos ciudades muy diferentes: La Coruña y Valencia. En La Coruña su menor masa crítica y la inferior valoración de la gestión urbana, se compensa por el elevado 
posicionamiento y el impulso de las grandes compañías privadas; en Valencia su posición refleja la política de marketing urbano de inversión intensiva pero con retorno económico menor de lo requerido para ajustar la relación coste-beneficio, y además la internacionalización de la imagen no ha ido acompañada de una internacionalización de su actividad empresarial (la principal compañía es de ámbito nacional en el sector de la distribución) capaz de compensar la crisis del sistema productivo tradicional, cuyo anterior dinamismo fue transferido a una excesiva exposición al "boom inmobiliario". Bilbao y Valencia son así, la antítesis, según el nivel de eficiencia, de un mismo modelo urbano.

- Clase II (entre 80 y 100): Mejor es la trayectoria de Málaga, que encabeza este grupo, convertida ya en el principal polo metropolitano de Andalucía y superando a Sevilla, que lo cierra. Su éxito se basa en un modelo importante de renovación y marketing urbano combinado con un proceso de innovación tecnológica que ha proporcionado un avance en empresas de nueva economía, gracias al buen resultado del parque tecnológico (el proyecto Málaga Valley) y en el posicionamiento como destino del turismo cultural. Completan este grupo San Sebastián, Pamplona y Oviedo-Ciudad Astur, las ciudades más equilibradas en gestión urbana y desarrollo económico, porque combinan su calidad urbana con un sistema productivo local que ha logrado mantenerse fuerte durante la crisis. Y lo cierra Sevilla, a pesar de su tamaño (es la cuarta área metropolitana de España) y de la importancia de las inversiones del sector público efectuadas anteriormente a la crisis (Expo 92); algunos sectores emergentes (renovables y aeronáutica) se han encontrado con importantes problemas de competitividad y no han logrado una reactivación que dotara a la economía local del impulso suficiente para superar el modelo clásico de ciudad de servicios y de turismo.

- Clase III (entre 60 y 80): En este grupo están seis ciudades Vigo, Zaragoza, Palma de Mallorca, Valladolid, Vitoria y Granada. De ellas, cuatro basan su economía en la industria del automóvil, en la biomédica (Granada) y dos en el turismo (Granada y Palma), tratándose en todos los casos de ciudades muy especializadas pero donde la iniciativa privada ha sido muy importante.

- Clase IV (menos de 60): Murcia, Santander, Alicante, Elche, Jerez y Cádiz. De las ciudades seleccionadas son estas las que ocupan el último lugar, y en todas ellas hay un factor común: una base económica muy polarizada y debilitada por el cambio del modelo productivo global, y posteriormente muy afectada por la crisis, lo cual acentuó su regresión. Una gestión urbana poco innovadora prolongó esta situación deficitaria.

\section{Las ciudades emprendedoras}

El índice de emprendimiento lo hemos estimado mediante el cálculo del cociente entre el índice de aportación privada y la iniciativa pública o institucional. Los resultados los exponemos agrupados en clases: 
- Clase I: Aquí están las ciudades donde el peso del sector privado es mayor, y son por orden: La Coruña $(1,16)$, donde el peso de la sede Inditex-Zara es mucho mayor que el de la propia ciudad en términos de tamaño y función; Palma de Mallorca $(1,01)$ por su fuerte desarrollo turístico-empresarial; Madrid $(1,00)$ la capital y principal sede en las grandes empresas. En las tres tiene importancia el sector financiero, cuya concentración en las dos mayores ciudades (Madrid y Barcelona) ha reforzado indirectamente, y en términos comparativos, el perfil económico de aquellas que han mantenido una sede bancaria regional, al mismo tiempo que se consolidaron otros factores estratégicos asociados a la nueva economía (Bilbao, La Coruña).

- Grupo II: Málaga $(0,95)$ y Vigo $(0,88)$ donde el desarrollo de empresas multinacionales del transporte y nuevos desarrollos tecnológicos han supuesto un notable impulso a la ciudad. En las siguientes ciudades la inversión pública ha superado a la privada aun cuando la actividad empresarial sea considerable: Valencia $(0,80)$, con la misma posición Bilbao $(0,78)$ y Zaragoza $(0,78)$, y finalmente Barcelona $(0,77)$ donde las acciones institucionales superan el impulso de las grandes empresas. Todas ellas tienen en común un posicionamiento fuerte de las instituciones y de las empresas en el desarrollo de la ciudad, con la asunción de nuevas funciones económicas en el comercio y el turismo internacional, y en la expansión de la industria automovilística y tecnológica preferentemente. Un caso singular es el de Elche pero ya hemos expuesto su situación y lo explicaremos cuando analicemos la posición de salida.

- Grupo III: Son nueve ciudades las que forman este grupo. Todas ellas tienen en común la estabilidad de su base económica, pero carecen del peso que aportan las grandes sedes empresariales y las actuaciones han estado orientadas más el urbanismo que la promoción económica de la ciudad. Son éstas: Sevilla $(0,75)$, Pamplona $(0,74)$ Vitoria $(0,72)$, Oviedo $(0,70)$, Valladolid y Santander en el mismo puesto $(0,68)$ Oviedo $(0,65)$, Alicante $(0,62)$ y San Sebastián $(0,61)$.

- Grupo IV: En el último grupo quedan tres ciudades donde la crisis del sistema productivo local ha producido un estancamiento o regresión de le economía urbana y que el turismo no ha podido compensar. Son Murcia $(0,60)$, Cádiz $(0,59)$ y Jerez $(0,39)$.

A la vista de estos resultados, y tal como nos habíamos propuesto en la hipótesis inicial, hicimos una comprobación estadística para saber si había una relación causal diferencial entre el peso de cada tipo de iniciativa y el valor total del índice de posicionamiento. El resultado no fue como esperábamos, ya que los coeficientes de correlación obtenidos, aunque consistentes, indican un peso semejante en cada una de las variables consideradas. En efecto, el coeficiente entre el peso de la iniciativa privada y el índice de posicionamiento fue de 0,97, mientras que el correspondiente a la iniciativa pública fue de 0,96, y aunque otorga un mayor papel a la iniciativa empresarial en el posicionamiento de las ciudades la diferencia es muy pequeña para poder considerarla significativa. 
Tabla 2: Correlación entre las variables y el total

\begin{tabular}{|l|l|}
\hline Coef. Correlación Privado-Total & 0,97 \\
\hline Coef. Correlación Público-Total & 0,96 \\
\hline
\end{tabular}

Fuente: elaboración propia

Sin embargo, al comparar estos resultados con el peso proporcional que cada tipo de iniciativa tiene en la formación del índice de posicionamiento, constatamos que las iniciativas privadas alcanzan un valor total de 764,11, y que su peso proporcional es del 43,45\% en el conjunto del sistema metropolitano considerado, mientras que las iniciativas públicas tienen un peso mayor $(994,44)$ y, en consecuencia, una proporción del $56,55 \%$, es decir mayor que la anterior.

Finalmente, haremos una última comparación, en este caso entre los coeficientes de participación en la formación del índice de posicionamiento y las correlaciones calculadas

Tabla 3. Comparativa

\begin{tabular}{|l|c|c|c|}
\hline & Privado & Público & Total \\
\hline Valor medio & 34,73182 & 45,15 & \\
\hline Suma & 764,1 & 993,3 & 1758 \\
\hline $\begin{array}{l}\text { Proporción en relación con el } \\
\text { total }\end{array}$ & 43,46664 & 56,5049 & \\
\hline
\end{tabular}

Fuente: elaboración propia

Este análisis nos aporta la siguiente conclusión: la iniciativa privada, a pesar de tener un peso menor que la pública en el posicionamiento de las ciudades, su correlación con el índice es ligeramente mayor, de lo cual se deduce que la iniciativa privada es más eficiente para el posicionamiento urbano que la pública, pero que, a la vista de las diferencias existentes, puede afirmarse que la combinación de la iniciativa pública y la privada es la estrategia más eficaz para el posicionamiento de las ciudades, tal como sostiene la teoría de la cooperación público-privada como estrategia de desarrollo territorial.

\section{Las ciudades con mejor situación de salida}

Como complemento de los análisis anteriores, y tal como enunciamos en los objetivos de esta investigación, haremos a continuación un análisis del índice de posicionamiento socioeconómico de las ciudades estudiadas a la salida del ciclo recesivo de la crisis económica, basándonos en los indicadores estadísticos anteriormente expuestos. Con ellos hemos realizado, en primer lugar, un estudio evolutivo del periodo 2007-2012, cuyos años marcan las fechas de máxima recesión y de inicio de la salida, es decir la tasa de incremento posicional. Los resultados están en la tabla siguiente. 


\subsection{Los incrementos posicionales 2007-2012}

Para empezar, calculamos los índices de correlación entre la tasa de crecimiento y los índices de posicionamiento anteriores, comprobando que en ninguno de los supuestos existe correlación significativa desde el punto de vista estadístico; por lo tanto su interpretación nos lleva a afirmar que las ciudades que experimentaron mayor crecimiento no fueron siempre aquellas donde los índices de posicionamiento anterior eran mayores, pudiendo entonces suponerse que dichos cambios se deberán a factores individuales asociados a factores externos.

Tabla 4. Correlaciones entre Índice evolutivo y privado, público y total

\begin{tabular}{|c|c|}
\hline Variable & Valor \\
\hline Privado & 0,002 \\
\hline Público & $-0,080$ \\
\hline Total & $-0,037$ \\
\hline
\end{tabular}

Fuente: elaboración propia a partir del INE, la Seguridad Social y el Anuario La Caixa

La explicación de estos resultados la podemos encontrar al analizar cada una de las ciudades, cuya relación completa figura en la tabla siguiente.

Tabla 5. Índice evolutivo: tasa de incremento posicional

\begin{tabular}{|l|c|}
\hline Ciudad & $\begin{array}{c}\text { Índice } \\
\text { Evolutivo }\end{array}$ \\
\hline Elche & 10,00 \\
\hline Pamplona & 9,00 \\
\hline San Sebastián & 8,76 \\
\hline Málaga & 8,15 \\
\hline Vitoria & 8,00 \\
\hline Oviedo & 7,83 \\
\hline Jerez & 6,90 \\
\hline Vigo & 6,64 \\
\hline Palma de Mallorca & 6,59 \\
\hline La Coruña & 6,28 \\
\hline Madrid & 6,02 \\
\hline Alicante & 5,14 \\
\hline Santander & 4,21 \\
\hline Granada & 4,18 \\
\hline Zaragoza & 4,07 \\
\hline Sevilla & 4,07 \\
\hline Barcelona & 3,48 \\
\hline Valencia & 3,21 \\
\hline Murcia & 2,25 \\
\hline
\end{tabular}


Tabla 5. Continuación

\begin{tabular}{|l|c|}
\hline Ciudad & $\begin{array}{c}\text { Índice } \\
\text { Evolutivo }\end{array}$ \\
\hline Bilbao & 1,11 \\
\hline Cádiz & 0,76 \\
\hline Valladolid & 0,00 \\
\hline
\end{tabular}

Fuente: elaboración propia a partir del INE, la Seguridad Social y el Anuario La Caixa

Esta es lo que hemos deducido de los datos:

1. Resulta llamativo, en principio, que sea Elche, una ciudad del último rango, y una de las que presenta valores más negativos en las variables económicas y en el resto de los ítems, la que experimente el mayor incremento. Lógicamente una situación así tendría que deberse a un factor nuevo de carácter exógeno; y, en parte, así fue. La causa, como ya anticipamos al principio, fue que la dinámica regresiva de la industria del calzado que constituía la base de la economía urbana, se recuperó con la implantación de una planta o unidad de producción del grupo Inditex que aportó un impulso notable al empleo y a las otras variables económicas relacionadas. De todos modos no fue una ruptura con el modelo anterior, porque fue el know how acumulado lo que determinó la instalación de la planta destinada a abastecer una nueva marca del grupo primero, y a las demás después de la gama de artículos de piel de la empresa coruñesa.

2. En un segundo grupo pueden incluirse cuatro ciudades: Pamplona, San Sebastián, Málaga y Vitoria. Las cuatro experimentaron una renovación expansiva del terciario productivo y del turismo urbano.

3. A continuación son seis las ciudades cuyo incremento oscila entre el $6 \%$ y el $8 \%$ : OviedoCiudad Astur, Jerez, Vigo, Palma de Mallorca, La Coruña y Madrid. Todas tienen en común una base industrial consolidada, un terciario empresarial y/o turístico, y una estructura económica estable. La única excepción es el caso de Jerez de la Frontera, que como antes Elche, es una de las peor posicionadas según todos los índices. El caso de Madrid puede deberse al efecto tamaño que siempre hace bajar las tasas de crecimiento, aunque también puede estar relacionado con el fuerte impacto de la crisis en el mercado de trabajo de la región metropolitana madrileña, aunque los índices empresariales y otros de tipo cualitativo sigan siendo superiores a los de otras ciudades.

4. Otras seis ciudades experimentaron un incremento intermedio (entre el $4 \%$ y el $6 \%$ ). Son: Alicante, Santander, Granada, Zaragoza, y Sevilla. Es difícil hacer un diagnóstico común, porque se trata de modelos diferentes, por eso la explicación que proponemos se basa en la relación de su base económica con la demanda interna, ya que son todas ciudades turísticas y 
de servicios, y por eso el cambio de coyuntura produjo una mejora de las variables que entran en el índice estimado.

5. Más interesante es la lectura de las ciudades que experimentaron un menor incremento. Por un lado está el caso de Madrid, a cuya posible explicación ya nos referimos antes, pero también figuran las tres grandes ciudades que en la fase anterior habían ostentado los puesto de cabeza en las inversiones del sector público y en las políticas estratégicas y de marketing urbano, como es el caso de Bilbao, Barcelona, Valencia; pero, en otros, se debe simplemente a economías urbanas estabilizadas en un sector no expansivo o regresivo (Cádiz y Murcia), o que se redujeron las inversiones privadas o parte de su producción se trasladó a otra ciudad (caso de Valladolid); aunque no descartamos el efecto de la política de austeridad del sector público que afectó principalmente a las capitales administrativas autonómicas, como son la mayoría de estas ciudades, que antes eran las más favorecidas por las iniciativas políticas. Una pluralidad de situaciones pero que todas tienen en común factores directamente relacionados con un modelo de economía urbana muy dependiente del sector público.

\subsection{Las ciudades más resistentes a la fase recesiva: el posicionamiento económico de salida}

Para terminar, presentamos la clasificación final de las ciudades atendiendo a su posicionamiento de salida, según el índice estructural de desarrollo urbano que hemos calculado utilizando las variables socioeconómicas expuestas en el apartado metodológico. Empezaremos, como siempre, calculando las correlaciones existentes. En este caso los resultados fueron más significativos que los anteriores, confirmando nuestra hipótesis inicial, como explicamos a continuación:

1. El coeficiente de correlación entre el índice de posicionamiento obtenido para el periodo recesivo y el índice de desarrollo urbano al inicio de la salida $(r=0.41)$ sin ser un valor especialmente alto es significativo.

2. Sin embargo, el valor más significativo $(r=0.49)$ corresponde al papel de la iniciativa privada, que supera notablemente al valor del coeficiente correspondiente al sector público $(r=0.30)$. Un resultado que confirma plenamente la hipótesis inicial según la cual el peso del sector privado en la mejora del posicionamiento económico de las ciudades a la salida de la fase recesiva de la crisis ha sido mayor que el que tuvo el sector público. Exactamente lo contrario que de lo que ocurría en la etapa anterior, cuando las grandes inversiones públicas, casi siempre asociadas a grandes eventos, o a grandes inversiones promocionales, eran las determinantes del desarrollo de la mayoría de las grandes ciudades (Barcelona, Sevilla, Valencia, Zaragoza, y en menor medida Bilbao). 
Tabla 6. Correlaciones entre el índice de desarrollo urbano y los índices de posicionamiento

\begin{tabular}{|c|c|c|}
\hline $\begin{array}{c}\text { Coef Correl } \\
\text { Privado }\end{array}$ & $\begin{array}{c}\text { Coef Correl } \\
\text { Público }\end{array}$ & $\begin{array}{c}\text { Coef Correl } \\
\text { Total }\end{array}$ \\
\hline 0,49 & 0,30 & 0,41 \\
\hline
\end{tabular}

Fuente: elaboración propia

La lectura de la tabla que contiene los resultados pormenorizados nos presenta una situación que poco tiene que ver con el posicionamiento de las ciudades en la etapa anterior, y que no permite establecer una relación lineal entre el peso de la iniciativa pública o de la privada y el índice de desarrollo urbano, ya que se mezclan ambas cosas, y encontramos ciudades correspondientes a cada uno de los tipos anteriormente estudiados, que ahora ocupan posiciones aparentemente aleatorias. De ahí que las correlaciones no sean significativas. Por eso, hemos procedido a tener en cuenta otros factores geográficos que parecen haber intervenido más directamente en el desarrollo urbano en la salida de la fase recesiva de la crisis.

Tabla 7. Índice de desarrollo urbano

\begin{tabular}{|l|c|}
\hline Ciudad & Índice D.U. \\
\hline Pamplona/Iruña & 10,0 \\
\hline Madrid & 9,8 \\
\hline Palma de Mallorca & 9,7 \\
\hline Vitoria-Gasteiz & 9,5 \\
\hline Coruña, A & 9,5 \\
\hline Barcelona & 8,7 \\
\hline Zaragoza & 6,8 \\
\hline Murcia & 6,6 \\
\hline Oviedo-C-Astur & 6,0 \\
\hline San Sebastián & 6,0 \\
\hline Alicante & 5,7 \\
\hline Valencia & 5,3 \\
\hline Santander & 5,3 \\
\hline Vigo & 5,2 \\
\hline Elche & 5,2 \\
\hline Bilbao & 4,5 \\
\hline Valladolid & 3,7 \\
\hline Málaga & 3,3 \\
\hline Sevilla & 3,0 \\
\hline Granada & 2,6 \\
\hline Jerez de la Frontera & 0,2 \\
\hline Cádiz & 0,0 \\
\hline
\end{tabular}

Fuente: elaboración propia a partir de INE y Eurostat 
1. Entre las diez primeras ciudades, cinco se sitúan en el norte de España, cuatro en el eje mediterráneo catalán-levantino y en el centro Madrid; por el contrario, los cinco últimos puestos están ocupados por las ciudades andaluzas. Si trazamos una diagonal entre La Coruña y Murcia, pasando por Madrid, nos encontramos que todas las ciudades mejor situadas están al norte de dicha diagonal, y en cambio, las peor posicionadas están al sur de esa diagonal.

2. Las ciudades con mayor calidad de vida, con una base productiva muy especializada y con una mayor internacionalización de su economía son las que ocupan los primeros lugares, lo cual coincide con la mayoría de las que antes presentaban una combinación más favorable entre lo privado y lo público y que, por eso encabezaban la lista de las ciudades más emprendedoras. A este modelo se ajustan las ciudades que ocupan los siete primeros lugares: Pamplona, Madrid, Palma, Vitoria, La Coruña y Zaragoza.

3. Es llamativo que las ciudades estrella de la renovación y el marketing urbano, y que también ocupaban lugares destacados como ciudades emprendedoras, bajen ahora a lugares medios, como Valencia o Bilbao, e incluso bajos, como Málaga. Podría pensarse que el modelo de marketing urbano no ha sido tan eficaz como parecía, y que solo ha funcionado cuando la ciudad, además de otras fortalezas, ha logrado posiciones preeminentes en el mercado turístico internacional (caso de Barcelona).

4. Otra consideración que podemos hacer es que las ciudades de base industrial intensiva en mano de obra han perdido posiciones, como ocurre con las ciudades del automóvil (Valladolid, Vigo y Valencia).

5. Unas pocas ciudades han tenido un impulso positivo, como ocurre con el caso comentado de Elche, pero salvo este los demás apenas han supuesto variaciones significativas.

\section{Conclusiones finales}

1. Digamos, en primer lugar, que -como ya hemos señalado- los resultados confirman la hipótesis inicial: el papel de la iniciativa empresarial en el posicionamiento post-recesión de las ciudades ha sido la más determinante.

2. Con todo, cuando hay una combinación equilibrada entre una gestión pública eficiente y una actividad empresarial competitiva la estabilidad del crecimiento es mayor. De hecho, las ciudades que en la fase recesiva presentaban un mejor balance público-privado también fueron las que mejor se situaron en el índice de desarrollo urbano en el cambio de coyuntura.

3. Las ciudades con mayor calidad de vida, con una base productiva especializada y una mayor internacionalización de su economía, son las que ocupan los primeros lugares. Casualmente, coincide con la mayoría de las ciudades que antes presentaban una combinación más favorable 
entre lo privado y lo público, y que, por eso encabezan ahora la lista de las ciudades más emprendedoras y expansivas.

4. Se acentuó el componente geográfico de desigualdad, y coincide con el diferente grado de desarrollo de las regiones en las que las ciudades se inscriben. Así, ya en la fase recesiva, en las ciudades de la mitad norte lo privado había sido más importante, mientras que en las ciudades de la mitad sur era lo público lo más determinante. Este factor se acentuó aún más en la situación de salida, ya que trazando una diagonal entre La Coruña y Murcia, pasando por Madrid, nos encontramos que todas las ciudades mejor situadas están al norte de dicha diagonal, y en cambio, las peor posicionadas están al sur de esa diagonal.

5. Es llamativo que las ciudades estrella de la renovación y el marketing urbano y que ocupaban lugares destacados en su posicionamiento urbano en la etapa anterior, bajen ahora a lugares medios, como Valencia o Bilbao, e incluso bajos, como Málaga. Podría pensarse que el modelo de marketing urbano no ha sido tan eficaz como parecía, y que solo ha funcionado cuando la ciudad añadía a la imagen otras fortalezas funcionales o económicas.

6. Todos los rankings presentan una importante divergencia entre el tamaño de las ciudades y los índices de situación y de posicionamiento. El caso más evidente es el de la ciudad de La Coruña, por el efecto sede de Inditex, pero también ocurre en ciudades medias como Pamplona, Vitoria y Palma de Mallorca. Todas ellas combinan el peso de lo privado, la calidad urbana y la especialización de los servicios.

7. Al comparar el peso proporcional de cada tipo de iniciativa en la formación del índice de posicionamiento, constatamos lo siguiente: que las iniciativas privadas, con un valor total de 764,11 puntos y un peso proporcional de $43.45 \%$, fue en la etapa recesiva menor que el de las iniciativas públicas (con un valor de 994,44 puntos y una proporción del 56-55\%). En cambio, si comparamos los coeficientes de participación en la formación del índice de posicionamiento y las correlaciones calculadas, comprobamos que en la iniciativa privada, a pesar de tener un peso menor que la pública, el valor de la correlación con el índice de desarrollo urbano final es mayor, es decir su contribución fue más consistente. Ambas constataciones demuestran que la iniciativa privada fue más efectiva para el posicionamiento urbano que la pública, pero así como en la fase recesiva el protagonismo de la inversión pública fue más importante, en la fase de salida fue la iniciativa privada la que más contribuyó a que las ciudades alcanzaran posiciones más competitivas.

8. Un último aspecto a considerar, aunque estadísticamente sea menos significativo que los anteriores, es que el modelo de desarrollo urbano español de la fase de crecimiento anterior a la crisis, caracterizado por la burbuja inmobiliaria, el excesivo gasto público y la política despilfarradora de los gobiernos autonómicos, ha dejado de ser efectivo, es más ha sido la 
causa de que muchas ciudades que obtuvieron un fuerte impulso en su índice de posicionamiento inicial hayan quedado postergadas en su posicionamiento urbano a la salida del ciclo recesivo de la crisis, porque los factores del anterior crecimiento fueron en parte los inductores de la crisis económica y lógicamente de la crisis del desarrollo urbano. Un caso expresivo es el de las ciudades que son capitales autonómicas y que en esta función tenían su principal fortaleza: todas ellas han perdido posiciones y competitividad.

No queremos terminar sin destacar que estas conclusiones presentan bastante coincidencia con las que en una investigación anterior obtuvimos cuando analizamos el posicionamiento de las ciudades europeas, en donde las ciudades medias que presentaban mayor calidad de vida, que eran sedes de multinacionales especializadas, que poseían una base financiera y que desarrollaban centros de I+D eran las mejor posicionadas para ser ciudades competitivas en el nuevo entorno económico global (Precedo, A. y Miguez, A., 2013). Esta misma situación se comprueba ahora para el caso español. 


\section{Bibliografía}

Belis, M. (2012). La ciudad, clave del siglo XXI. En M. Belil, J. Borja y M. Corti (Eds.), Ciudades, una ecuación imposible (pp. 11-22). Barcelona: Icaria Editorial.

Bellet, C., y Llop, J. M. (2004). Miradas a otros espacios urbanos: las ciudades intermedias. Scripta Nova, 8 165).

Burriel, E. L. (2008). La década prodigiosa del urbanismo español (1997-2006). Scripta Nova, 12(270).

Campos, Á. P., y Navarro, M. L. H. (2013). España ante la Gran Recesión del siglo XXI.

M@ppemonde, 111. Recuperado de

http://mappemonde-archive.mgm.fr/num39/articles/art13301_es.html

Clayton, N., y Morris, K. (2010). Recession, recovery and medium-sized cities. Londres: The Work Foundation.

Del Romero, L. (2010). Dos décadas de urbanismo-espectáculo en España: Los grandes eventos como motor de cambio urbano. Boletín de la Asociación de Geógrafos Españoles, 53, 309-327.

Florida, R. (2011). El gran reset. Barcelona: Paidós.

Folke, C. (2006). Resilience: the emergence of a perspective for socio-ecological systems analyses. Global Environmental Change, 16(3), 253-267.

García, M. (2010). The breakdown of the Spanish urban growth model: social and territorial effects of the global crisis. International Journal of Urban and Regional Research, 34(4), 967-980.

Gentier, A. (2012). Spanish Banks and the Housing Crisis: Worse than the Subprime Crisis? International Journal of Business, 17(4), 342-351.

Glaeser, E. (2011). El triunfo de las ciudades. Madrid: Taurus.

Lee, N., Morris, K., y Jones, A. (2009). How UK cities can respond and drive the recovery. Londres: The Work Foundation.

Méndez, R. (2012). Ciudades y metáforas. Sobre el concepto de resiliencia urbana. Ciudad y Territorio: Estudios Territoriales, 172, 215-232.

Méndez, R. (2012b). Ciudades, crisis y desempleo en España. Revista de Estudios de la Fundación $1^{\circ}$ de Mayo, 43, 13-15.

Méndez, R. (2013): Las escalas de la crisis: ciudades y desempleo en España. Madrid: Fundación $1^{\circ}$ de Mayo, Colección Estudios, n 60.

Méndez, R. (Dir.) (2010). Estrategias de innovación industrial y desarrollo económico en las ciudades intermedias de España 2010). Madrid: Fundación BBVA. 
Méndez, R., y Plaza, J. (2016). Crisis inmobiliaria y desahucios hipotecarios en España: Una perspectiva geográfica. Boletín de la Asociación de Geógrafos Españoles, 71, 99-127.

Méndez, R., Echaves, C., y Abad, L. (2015). Atlas de la crisis. Madrid: Tirant Humanidades.

Míguez, A. (2011). Ciudades intermedias y Calidad de vida. Santiago de Compostela: Ed. USC.

Míguez, A., y Precedo, A. (2008). De las áreas metropolitanas a las regiones urbanas. Modelos de gobernabilidad. Presentado en el XI Coloquio Ibérico. Alcalá de Henares-Pastrana, octubre de 2008.

Naredo, J. M. (2009). La cara oculta de la crisis. El fin del boom inmobiliario y sus consecuencias. Revista de Economía Crítica, 7, 313-340.

Navarro, C., Rodríguez, M. J., Herrera, M. R., y Mateos, C. (2012). Gobernabilidad y crisis urbana. El impacto de la crisis sobre las grandes ciudades españolas. Presentado en el XI Congreso Español de Sociología. Madrid, 10-12 Julio de 2013. Recuperado de http://www.fessociologia.com/files/congress/11/papers/268.pdf

Precedo A. (1996). Ciudad y Desarrollo Urbano. Madrid: Síntesis.

Precedo Ledo, A., y Miguez Iglesias, A. (2014). Una radiografía de las ciudades españolas. En R. B. Lois y A. Miramontes (Coords.), Reflexiones sobre las ciudades y el sistema urbano en tiempos de crisis (pp. 15-72). Santiago de Compostela: Tórculo de Ediciones.

Precedo Ledo, A., Orosa González, J. J., y Míguez Iglesias, A. (2010). De la planificación estratégica al marketing urbano: hacia la ciudad inmaterial. EURE, 36(108), 5-27.

Precedo, A. (2010). Un ensayo sobre la evolución de la geografía urbana en España: reinventar el estudio de la ciudad. Geographicalia, 58, 5-27

Precedo, A., y Míguez, A. (2008). La metropolización como estrategia de competitividad para las ciudades medias: el caso de Galicia. Presentado en el XI Coloquio Ibérico. Alcalá de HenaresPastrana, octubre de 2008.

Precedo, A. y Míguez, A. (2012). El sistema metropolitano de Galicia. De las Áreas Metropolitanas a las Regiones Urbanas. Ed. USC. Recuperado de http://hdl.handle.net/10347/6219

Precedo, A., y Míguez, A. (2013). Las Ciudades Medias en la globalización. Madrid: Síntesis.

Precedo, A., y Míguez, A. (2007). La actividad turística como factor de desarrollo en el sistema español de asentamientos (1997-2002). Boletín de la Asociación de Geógrafos Españoles, 45, 191-211. 
Precedo, A., Míguez, A., y Orosa, J. (2012). La calidad de vida de las ciudades gallegas: una aplicación empírica. Boletín de la Asociación de Geógrafos Españoles, 59, 275-300.

Romero, J. (2010). Construcción residencial y gobierno del territorio en España. De la burbuja especulativa a la recesión. Causas y consecuencias. Cuadernos Geográficos, 47, 17-46.

Romero, J., Jiménez, F., y Villoria, M. (2012). Unsustainable territories: causes of the speculative bubble in Spain (1996-2010) and its territorial, environmental and sociopolitical consequences. Environment and Planning C, 30, 467-486.

Vinuesa, J. (2013). El festín de la vivienda. Auge y caída del negocio inmobiliario en España. Madrid: Díaz \& Pons.

Vinuesa, J., y Martín, B. (2013). La (sobre)dimensió del creixement residencial a Madrid.

planejament urbanístic com a coartada. Documents d'Anàlisi Geogràfica, 59(1), 51-74. http://www.raco.cat/index.php/DocumentsAnalisi/article/view/262782/350257

Vono, D., y Bayona, J. (2011). El asentamiento residencial de los latinoamericanos en las principales ciudades españolas (2001-2009). Notas de Población, 91, 129-159. 